\title{
Distributed Coordinated Voltage Control for Distribution Networks with DG and OLTC based on MPC and Gradient Projection
}

Jiao, Wenshu; Chen, Jian; Wu, Qiuwei; Li , Canbing; Zhou, Bin; Huang, Sheng

Published in:

IEEE Transactions on Power Systems

Link to article, DOI:

10.1109/TPWRS.2021.3095523

Publication date:

2021

Document Version

Peer reviewed version

Link back to DTU Orbit

Citation (APA):

Jiao, W., Chen, J., Wu, Q., Li , C., Zhou, B., \& Huang, S. (2021). Distributed Coordinated Voltage Control for Distribution Networks with DG and OLTC based on MPC and Gradient Projection. IEEE Transactions on Power Systems, 37(1), 680 - 690. https://doi.org/10.1109/TPWRS.2021.3095523

\section{General rights}

Copyright and moral rights for the publications made accessible in the public portal are retained by the authors and/or other copyright owners and it is a condition of accessing publications that users recognise and abide by the legal requirements associated with these rights.

- Users may download and print one copy of any publication from the public portal for the purpose of private study or research.

- You may not further distribute the material or use it for any profit-making activity or commercial gain

- You may freely distribute the URL identifying the publication in the public portal 


\title{
Distributed Coordinated Voltage Control for Distribution Networks with DG and OLTC based on MPC and Gradient Projection
}

\author{
Wenshu Jiao, Jian Chen, Qiuwei Wu, Senior Member, IEEE, Canbing Li, Senior Member, IEEE, Bin \\ Zhou, Senior Member, IEEE, Sheng Huang
}

\begin{abstract}
This paper proposes a distributed coordinated voltage control scheme for distribution networks with distributed generation (DG) and on-load tap changer (OLTC). In this scheme, static synchronous compensators (STATCOMs), DG units and OLTC are coordinated to regulate voltages of all buses to be close to the nominal value in the distribution network, mitigate voltage fluctuations, and minimize the number of operations of OLTC while considering different temporal characteristics of voltage regulation devices. The optimization problem of coordinating DG units and STATCOMs is decomposed by the gradient projection (GP) method. The local controller optimizes the reactive power outputs of DGs and STATCOMs according to local voltage and reactive power measurements, and still achieves the optimal coordination of DG units and STATCOMS in a decentralized manner without a central controller or communication between local controllers. The OLTC control scheme is designed to correct the long-term voltage deviations based on model predictive control (MPC) while minimizing the number of operations. The local controllers send the calculated reactive power references of DG and STATCOMs to the OLTC controller, which achieves distributed coordinated voltage control and mitigates the computation burden. A distribution network with two $20 \mathrm{kV}$ feeders and $8 \mathrm{DG}$ units was used to validate the control performance of the proposed coordinated voltage control scheme.
\end{abstract}

Index Terms-Distributed control, distribution network, gradient projection (GP), model predictive control (MPC), voltage control.

\section{INTRODUCTION}

$\mathrm{T}$ HE increasing penetration of distributed generation in distribution networks has changed the power flow from traditional unidirectional to bidirectional [1]. The conventional passive distribution networks are gradually transforming into active distribution networks (ADNs) [2]. As the main element of ADNs, distributed generation (DG) such as photovoltaic (PV) and wind power can meet the increasing energy demand and alleviate the environmental issue. However, due to the

This work was supported by the National Natural Science Foundation of China under grant no. 51877124. Corresponding authors: Jian Chen (ejchen@sdu.edu.cn), Qiuwei Wu (qw@elektro.dtu.dk).

J. Wen and J. Chen are with the School of Electrical Engineering, Shandong University, Jinan 250061, China (e-mail: jknone@163.com, ejchen@sdu.edu.cn).

Q. Wu and S. Huang are with the Center for Electric Power and Energy, Department of Electrical Engineering, Technical University of Denmark (DTU), Kongens Lyngby2800, Denmark (e-mail: qw@elektro.dtu.dk, ).

C. $\mathrm{Li}$ is with the School of Electronic Information and Electrical Engineering, Shanghai Jiaotong University, Shanghai 200240, China (e-mail: licanbing@sjtu.edu.cn).

B. Zhou is with College of Electrical and Information Engineering, Hunan University, Changsha 410082, China (binzhou@hnu.edu.cn). high penetration of $\mathrm{DG}$, it brings several technical challenges such as voltage rise, power quality, stability and protection [3].

Voltage regulation is one of the key aspects for secure operation of ADNs. Maintaining the steady state voltage within permissible limits as well as ensuring voltage quality in the system are essential for the operation of ADNs. Conventional voltage regulation devices including on-load tap changer (OLTC), step voltage regulator (SVR), and switched capacitor banks (CBs) have the characteristics of slow response and discrete actions [4]. To better control the voltage, several advanced devices such as modern power electronics converts and STATCOMs are designed [5]. Consequently, in order to coordinate these voltage regulation devices, more effective control methods are urgently needed [6], which motivates a lot of research in this area.

Traditionally, voltage control methods are designed in a decentralized manner without any coordination between voltage regulation devices and DG units. Power factor control (PFC) is widely used in ADNs to ensure that reactive power output of DG tracks its active power output [7]. Through power factor control, DG units are locally controlled to mitigate the voltage rise in distribution networks [8]. Moreover, PFC is sometimes combined with voltage control, which is known as power factor control-voltage control (PFCVC) [9]. The OLTC transformers are usually controlled automatically to increase or decrease voltage by detecting whether the secondary voltage is within the specified range [10]. These traditional decentralized methods mentioned above are simple and of low-cost. However, they are often unable to achieve optimal control.

With the deployment of more control equipment, distribution networks become more complex and active. Therefore, the coordinated voltage control strategies have been developed with different levels of communication requirement and complexity. These schemes require a central controller to solve an optimization problem and regulate the entire network [5]. In [11], the optimal power flow (OPF) based voltage control scheme was designed, which aims to minimize the curtailed active power of DG units. In [12], the central controller was used to optimize parameters of piecewise linear functions and control the power output of PV units. A coordinated voltage regulation scheme combining SVR and OLTC was proposed for ADNs in [13]. In [14], a particle swarm optimization (PSO) algorithm was used to solve the multi-objective mixed-integer nonlinear programming 
(MINLP) problem. Centralized control methods are also used for real-time demand response [15], model predictive control [16] and model-free centralized control [17]. A combined local and centralized voltage control scheme was proposed in [18]. The reactive power of DG units is controlled locally according to a piecewise linear static $V-Q$ characteristic, and the central controller computes reactive power regulations based on MPC. In [19], an MPC-based framework was proposed to realize the local control of DG units, and the alternating direction method of multipliers (ADMM) algorithm was adopted to obtain the near-global optimization of voltage control. Considering uncertainties from DG units and demands, a robust constrained model predictive control (RCMPC) was formulated for centralized voltage control in [20]. These centralized methods usually result in a heavy computational burden for large-scale distribution networks. Therefore, distributed control schemes have attracted a lot of attention [21].

In the last few years, a lot of efforts have been made to demonstrate how the distributed control scheme can be implemented in distribution systems [22]. In [23] and [24], optimal distributed control strategies based on alternating direction method of multipliers (ADMM) and Distributed MPC were proposed, which reduce the voltage deviation by optimizing the reactive power output of DG. The effect of active power output of DG on voltage regulation is considered in [25]. In order to coordinate with OLTC, a communication based distributed control for OLTC and DG was proposed in [26] and [27]. The coordination by communication is proposed to avoid interference between the autonomous operations of different controllers. Distributed voltage control of distribution networks is summarized and compared in [28]. These methods mainly focus on optimizing the reactive power output of DG, and OLTC controller generally adopts line drop compensation (LDC) control. A weighted average of estimated voltage at all buses was used to control the OLTC in [29]. In [30] and [31], a two-stage voltage control strategy was proposed to coordinated DG units and OLTC. First, the OLTC operation is scheduled, and then the reactive power outputs of DG units are controlled. The existing methods do not consider the effect of fast var device when controlling OLTC, which cannot realize optimal coordination.

Therefore, this paper proposes a distributed coordinated voltage control for distribution networks based MPC and GP method with DG and OLTC. In this scheme, the reactive power outputs of DG units and STATCOMs, and switching operation of OLTC are optimally coordinated. Compared with the existing studies, the main contributions of the paper are summarized as follows:

1) Propose a distributed coordinated voltage control for distribution networks with DG and OLTC based on MPC and GP which can optimally control the reactive power outputs of DGs and STATCOMs without a central controller or communication between local controllers in the fast time scale and minimize the switching operation of OLTC with slow dynamics.

2) Develop a novel OLTC control method based on MPC which optimizes the coordination between the fast var devices and OLTC control and minimizes the switching operation time of the OLTC.

3) Design a unidirectional communication based coordination mechanism between the fast var devices and OLTC which is robust to the communication failure.

This paper is organized as follows. In Section II, the configuration of the framework of the distributed control scheme are presented. Decentralized control based on the GP method for DG and STATCOM is presented in Section III. The OLTC control based on MPC is described in Section IV. The simulation results are presented and discussed in Section $\mathrm{V}$, followed by conclusions.

\section{Overview of Distributed CoORdinated Voltage CONTROL SCHEME}

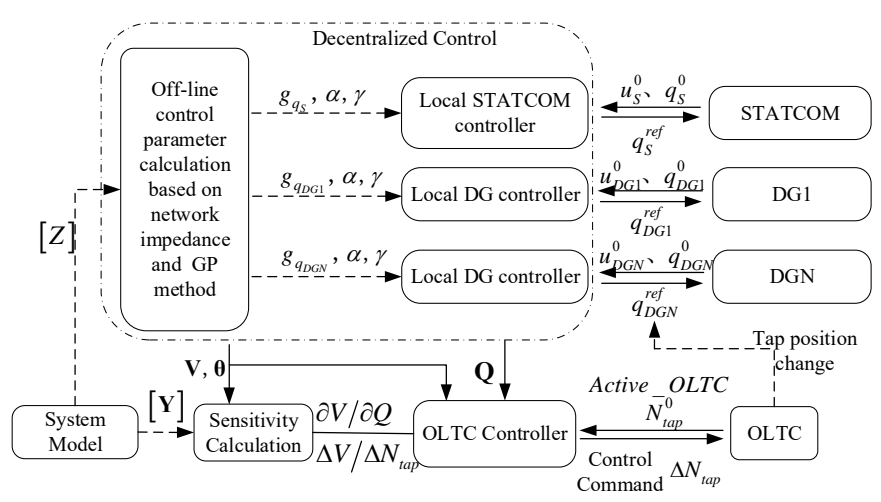

Fig. 1. Structure of distributed coordinated voltage control scheme.

Fig. 1 shows the structure of the proposed distributed coordinated voltage control scheme. The control scheme consists of two parts: decentralized control of DG and STATCOM and OLTC control, which considers different temporal characteristics of voltage control devices. Local controllers calculate the optimal reactive power outputs of STATCOM and DG units in a decentralized manner based on the GP method. According to the network impedance and GP method, the control parameters required for decentralized calculation such as the weight $\left(g_{q_{D C i}}, g_{q_{S}}\right)$ and step size $(\alpha, \gamma)$ are obtained by offline calculation. Combined with the measured local voltage $u_{i}^{0}$ and current reactive power output $q_{i}^{0}$, the optimal reactive power outputs $q_{i}^{\text {ref }}$ of DG and STATCOM are calculated. For the control of OLTC, the OLTC controller collects the network operation information including: 1) voltages of the critical buses selected according to the electrical distance [32];2) reactive power outputs calculated by the local controllers; and 3) operation information of OLTC transformers. Accordingly, the optimal action of the OLTC transformer that minimizes the voltage fluctuation of the entire distribution network is calculated based on the MPC method. The voltage sensitivity is calculated using the analytical method in [33] for the OLTC control. In addition, in order to reduce unnecessary operation of the OLTC, the optimal action command will not be sent to the OLTC until it satisfies certain conditions, which is described in Section IV. 
Both the decentralized control and OLTC control aim to keep the network voltage close to the nominal value. The tap position change of the OLTC can affect the voltage at each bus. Local controllers send reactive power outputs of DG units and STATCOM to the OLTC controller for computing the optimal operation of the OLTC. As such, the DG units, STATCOM and OLTC are coordinated to maintain the voltages within distribution networks to be close to the nominal value, mitigate voltage fluctuations, and minimize the switching operation of the OLTC.

\section{GP-BASEd DeCentralized Voltage CONTROL}

In this section, a GP-based decentralized voltage control is proposed for minimizing the voltage deviation from the nominal value within distribution networks in a decentralized way without loss of optimality of the optimal control problem. The reactive power outputs of DG units and STATCOM are optimized in the proposed decentralized control scheme. The optimality and stability analysis of the proposed solution method is presented in Appendix.

\section{A. Network Model}

The typical distribution network has a radial topology. Thus, the DistFlow model is used to describe the relationship between power flow and bus voltage [34].

$$
\begin{gathered}
P_{i j}=\sum_{k \in N_{j}} P_{j k}-p_{j}+r_{i j} \frac{P_{i j}^{2}+Q_{i j}^{2}}{\left|V_{i}\right|^{2}} \\
Q_{i j}=\sum_{k \in N_{j}} Q_{j k}-q_{j}+x_{i j} \frac{P_{i j}^{2}+Q_{i j}^{2}}{\left|V_{i}\right|^{2}} \\
\left|V_{i}\right|^{2}=\left|V_{j}\right|^{2}+2\left(r_{i j} P_{i j}+x_{i j} Q_{i j}\right)-\left(r_{i j}{ }^{2}+x_{i j}{ }^{2}\right) \frac{P_{i j}{ }^{2}+Q_{i j}{ }^{2}}{\left|V_{i}\right|^{2}}
\end{gathered}
$$

where $p_{j}$ and $q_{j}$ denote the injection of active and reactive power of node $j$; subscript $i j$ indicates that nodes $i$ and $j$ are adjacent, $r_{i j}$ and $x_{i j}$ denote the impedance of the line $i j$; $P_{i j}$ and $Q_{i j}$ denote the active and reactive power transported from node $i$ to node $j ; V_{i}$ denotes the voltage magnitude of node $i$; and $N_{j}$ is the set of nodes that are directly connected to node $j$.

The nonlinear term $\left(P_{i j}{ }^{2}+Q_{i j}{ }^{2}\right) /\left|V_{i}\right|^{2}$ denotes the squared line current magnitude, which represents the line power loss of the distribution network. Assuming the loss is negligible compared to line flow, model (1)-(3) can be simplified as a linearized model. The approximation error introduced is relatively small, at the order of $1 \%$ [35]. Let $N$ be the number of the distribution network nodes, and define the incidence matrix $\overline{\mathbf{A}} \in\{0, \pm 1\}^{N \times(N+1)}=\left[\mathrm{A}_{0}, \mathbf{A}^{\mathrm{T}}\right]^{\mathrm{T}}$ (let vector $\mathrm{A}_{0}^{\mathrm{T}}$ denote the first row of matrix $\overline{\mathbf{A}}$, and the rest of matrix denoted by $\mathbf{A}$ of size $N \times N$ ) as,

$$
\overline{\mathbf{A}}_{i j}=\left\{\begin{array}{c}
1, \text { if node } i \text { of } e_{i j} \text { is closer to slack bus } \\
-1, \text { if node } \text { i of } e_{i j} \text { is away from slack bus } \\
0, \quad \text { otherwise }
\end{array}\right.
$$

Based on this, the linearized model can be transformed into a matrix form,

$$
-\mathbf{A P}=-\mathbf{p}
$$

$$
\begin{gathered}
-\mathbf{A Q}=-\mathbf{q} \\
\overline{\mathbf{A}}^{\mathbf{T}}\left[U_{\mathrm{s}}, \mathbf{U}^{\mathbf{T}}\right]^{\mathrm{T}}=2(\mathbf{R P}+\mathbf{X Q})
\end{gathered}
$$

where $\mathbf{U}^{\mathbf{T}}$ are vectors of node voltage $U_{i}\left(U_{i}\right.$ denotes $\left.\left|V_{i}\right|^{2}\right)$, $U_{s}$ denotes the voltage of slack bus; $\mathbf{R}$ and $\mathbf{X}$ are diagonal matrix ( $r_{i j}$ and $x_{i j}$ are the diagonal elements, respectively); $\mathbf{P}$ $, \mathbf{Q}, \mathbf{p}$ and $\mathbf{q}$ are vectors corresponding to the matrix $\overline{\mathbf{A}}$. Substituting (5) and (6) into (7) yields,

$$
\mathbf{U}=2 \mathbf{A}^{-\mathrm{T}} \mathbf{R} \mathbf{A}^{-1} \mathbf{p}+2 \mathbf{A}^{-\mathrm{T}} \mathbf{X} \mathbf{A}^{-1} \mathbf{q}-\mathbf{A}^{-\mathrm{T}} \mathbf{A}_{0} \mathbf{U}_{\mathrm{s}}=\mathbf{M q}+\overline{\mathbf{U}}
$$

where $\mathbf{M}=2 \mathbf{A}^{-\mathrm{T}} \mathbf{X} \mathbf{A}^{-1}, \overline{\mathbf{U}}:=2 \mathbf{A}^{-\mathrm{T}} \mathbf{R} \mathbf{A}^{-1} \mathbf{p}-\mathbf{A}^{-\mathrm{T}} \mathbf{A}_{0} U_{\mathrm{s}}$. In [34], both $\mathbf{A}^{-\mathrm{T}} \mathbf{R} \mathbf{A}^{-1}$ and $\mathbf{A}^{-\mathrm{T}} \mathbf{X} \mathbf{A}^{-1}$ are proofed to be positive definite (PD).

\section{B. Mathematical Formulation of Voltage Control Problem}

1) Objective function: In order to resolve the voltage fluctuation problem of the active distribution network with DG units, the main objective of the decentralized control scheme is to keep the voltage level of the ADN close to the nominal value. The reactive power outputs of DGs and STATCOM are used to regulate voltages. Accordingly, the cost function is expressed as,

$$
\min _{\mathbf{q}} f(\mathbf{q})=\frac{1}{2}\|\mathbf{U}-\mathbf{U} \mathbf{r}\|_{\mathbf{G}_{U}}^{2}
$$

where $\|\mathbf{U}-\mathbf{U r}\|_{\mathbf{G}_{U}}^{2}=(\mathbf{U}-\mathbf{U r})^{\mathrm{T}} \mathbf{G}_{U}(\mathbf{U}-\mathbf{U r}) ; \mathbf{q}$ denotes the vector of Var output; $\mathbf{U}_{r}$ is the voltage reference, which is set to "1" in this paper; and $\mathbf{G}_{U}$ is the weight matrix of the voltage fluctuation.

2) Constraints: Consider the reactive power output limit of the DG units and STATCOM, the available reactive power constraint is,

$$
\mathbf{q}^{\min } \leq \mathbf{q} \leq \mathbf{q}^{\max }
$$

where $\mathbf{q}^{\min }$ and $\mathbf{q}^{\max }$ denote the lower and upper limits of the reactive output.

For the distribution network, the equality constraint is,

$$
\mathbf{U}=\mathbf{M q}+\overline{\mathbf{U}}
$$

3) Decentralized voltage control based on GP: The optimal problem (9) can be reformulated as a standard quadratic programming problem, which can be efficiently solved in a centralized method. However, due to the high penetration of DG units, centralized control will have a huge computation burden. Thus, a decentralized control scheme based on the GP is used for DG and STATCOM controllers.

The optimal solution of the optimization problem (9)-(11) can be iteratively obtained along the negative gradient direction. Moreover, the solution after each iteration should satisfy the inequality constraint (10). Therefore, a general form of the solution is,

$$
\mathbf{q}(k+1)=\mathbf{q}(k)+\alpha\left([\mathbf{q}(k)-\gamma \nabla f(\mathbf{q}(k))]^{+}-\mathbf{q}(k)\right)
$$

where $k$ is the number of iterations; $\alpha \in(0,1]$ and $\gamma>0$ are the step size control parameters, which ensures that the iteration solution satisfies the constraints (9) as long as the initial value satisfies the constraint; $\mathbf{z}$ denotes a column vector, $[\mathbf{z}]^{+}$denotes a method that maps to a constraint set, and its value can be defined as, 


$$
\left[\mathrm{z}_{i}\right]^{+}= \begin{cases}\mathrm{q}_{i}^{\min }, & \text { if } \mathrm{z}_{i}<\mathrm{q}_{i}^{\min } \\ \mathrm{Z}_{i}, & \text { if } \mathrm{q}_{i}^{\min }<\mathrm{z}_{i}<\mathrm{q}_{i}^{\max } \\ \mathrm{q}_{i}^{\max }, & \text { if } \mathrm{z}_{i}>\mathrm{q}_{i}^{\max }\end{cases}
$$

The basic GP method converges slowly. To improve the convergence performance, a scaled GP method is proposed. Firstly, introduce a scaled matrix $\mathbf{H}$ that is positive and transform the optimal problem using the rules $\mathbf{q}_{H}:=\mathbf{H}^{1 / 2} \mathbf{q}$,

$$
\begin{aligned}
& \min _{\mathbf{q}_{\mathbf{H}}} H\left(\mathbf{q}_{H}\right) \equiv f\left(\mathbf{H}^{1 / 2} \mathbf{q}_{\mathbf{H}}\right) \\
& \text { subject to } \mathbf{q}^{\min } \leq \mathbf{H}^{-1 / 2} \mathbf{q}_{\mathbf{H}} \leq \mathbf{q}^{\max } \\
& \mathbf{U}=\mathbf{M}\left(\mathbf{H}^{-1 / 2} \mathbf{q}_{\mathbf{H}}\right)+\overline{\mathbf{U}}
\end{aligned}
$$

The iteration equation after the transformation becomes,

$$
\mathbf{q}_{H}(k+1)=\mathbf{q}_{H}(k)+\alpha\left(\left[\mathbf{q}_{H}(k)-\gamma \nabla H\left(\mathbf{q}_{H}(k)\right)\right]^{+}-\mathbf{q}_{H}(k)\right)(17)
$$

Considering the rules $\nabla H\left(\mathbf{q}_{H}(k)\right)=\mathbf{H}^{-1 / 2} \nabla f(\mathbf{q}(k))$, $\left[\mathbf{q}(k)-\gamma \mathbf{H}^{-1} \nabla f(\mathbf{q}(k))\right]^{+}=\mathbf{H}^{-1 / 2}\left[\mathbf{q}_{H}(k)-\gamma \nabla H\left(\mathbf{q}_{H}(k)\right)\right]^{+}$and $\mathbf{q}(k)=\mathbf{H}^{-1 / 2} \mathbf{q}_{H}(k)$, the iteration equation can be written as,

$$
\mathbf{q}(k+1)=\mathbf{q}(k)+\alpha\left(\left[\mathbf{q}(k)-\gamma \mathbf{H}^{-1} \nabla f(\mathbf{q}(k))\right]^{+}-\mathbf{q}(k)\right)
$$

Eq. (18) is the final form of the iteration equation of the scaled GP method. In this way, the convergence rate of the algorithm depends on the maximum and minimum eigenvalues of its Hessian $\mathbf{H}^{-1 / 2} \nabla^{2} f(\mathbf{q}) \mathbf{H}^{-1 / 2}$.The closer the eigenvalues, the faster the convergence rate. Thus, the value of $\mathbf{H}$ should be close to $\nabla^{2} f(\mathbf{q})$ in order to improve the convergence performance.

As shown in (18), the global gradient information $\nabla f(\mathbf{q}(k))$ is necessary for each iteration. In order to obtain the optimal solution in a decentralized way, the gradient information should be decoupled and be measured locally. For the objective function in (9), its gradient $\nabla f(\mathbf{q}(k))$ is calculated as,

$$
\nabla f(\mathbf{q}(k))=\left(\mathbf{M}^{\mathrm{T}} \mathbf{G}_{U} \mathbf{M}\right) \mathbf{q}(k)+\mathbf{M} \mathbf{G}_{U}\left(\overline{\mathbf{U}}-\mathbf{U}_{\mathrm{r}}\right)
$$

By taking $\mathbf{G}_{U}=\mathbf{M}^{-1}$, the gradient can be transformed into,

$$
\nabla f(\mathbf{q}(k))=\mathbf{M q}(k)+\overline{\mathbf{U}}-\mathbf{U}_{\mathrm{r}}=\mathbf{U}(k)-\mathbf{U}_{\mathrm{r}}
$$

where $\mathbf{U}(k)$ denotes a vector of the voltage at each node at the $k$ th iteration. In this way, the global gradient information can be calculated by the local voltage and reactive power output. This is also the key to the decomposition of iterations, i.e., local measurements can reflect the global gradient information.

Moreover, the scaled matrix $\mathbf{H}$ should also be a diagonal matrix for the complete decomposition of the iteration equation. Considering $\mathbf{H}$ should be close to $\nabla^{2} f(\mathbf{q})$, a diagonal approximation to the Hessian matrix is used, i.e.,

$$
\mathbf{H}=\operatorname{diag}\left(\mathrm{h}_{1}, \mathrm{~h}_{2}, \ldots \mathrm{h}_{\mathrm{N}}\right)=\left[\begin{array}{lll}
{\left[\nabla^{2} f(\mathbf{q})\right]_{11}} & & \\
& \ddots & \\
& & {\left[\nabla^{2} f(\mathbf{q})\right]_{N N}}
\end{array}\right]
$$

where $\nabla^{2} f(\mathbf{q})=\mathbf{M}$ and $\mathbf{H}$ are positive definite.

Finally, the iteration equation of the decentralized reactive power control is transformed as,

$$
q_{i}(k+1)=q_{i}(k)+\alpha\left(\left[q_{i}(k)-\gamma h_{i}^{-1}\left(U_{i}(k)-U_{r}\right)\right]^{+}-q_{i}(k)\right)(22
$$

\section{OLTC CONTROL SCHEME BASED ON MPC}

An MPC-based OLTC control scheme is proposed in this section to determine the optimal action of the OLTC considering the network and coordination with local voltage controllers of DG units and STATCOM.

\section{A. Predictive Model}

1) DG units and STATCOM: The inverter-based DG units with short response time is used in this paper. Based on this, for the decentralized reactive power control scheme mentioned in Section III, the reactive power outputs of DG and STATCOM in the predicted range can be obtained as,

$$
\mathbf{Q}^{P}[k]=\left[q_{S}(k), q_{1}(k) \ldots, q_{N}(k)\right]^{\mathrm{T}}
$$

where $\mathbf{Q}^{P}[k]$ denotes the reactive power injection for the $k$ th prediction step. Eq. (23) is derived from (22).

2) Network: Generally, power flow equations are used to model the distribution network as (1)-(3). In this subsection, a model derived from the Taylor approximation at the real-time operating point is adopted to predict the voltage of the network, which is,

$$
\mathbf{V}^{P}=\mathbf{V}_{0}+\frac{\partial \mathbf{V}}{\partial \mathbf{Q}^{\mathrm{T}}} \Delta \mathbf{Q}+\frac{\partial \mathbf{V}}{\partial N_{\text {tap }}} \Delta N_{\text {tap }}+\frac{\partial \mathbf{V}}{\partial \mathbf{P}_{D G}^{\mathrm{T}}} \Delta \mathbf{P}
$$

where $\mathbf{V}^{P}$ denotes the predictive voltage; $\partial \mathbf{V} / \partial \mathbf{P}_{D G}^{\mathrm{T}}$, $\partial \mathbf{V} / \partial \mathbf{Q}^{\mathrm{T}}$ and $\partial \mathbf{V} / \partial N_{\text {tap }}$ denote the voltage sensitivities with respect to the changes of active power injection, reactive power output and OLTC tap position; and $\Delta N_{\text {tap }}$ and $\Delta \mathbf{P}$ are the variation of OLTC tap position and active power injection.

\section{B. Sensitivity Calculation and Critical Bus Selection}

\section{1) Sensitivity calculation:}

The voltage sensitivity calculation is necessary to develop the linearized network model mentioned above for voltage prediction. Traditional methods for voltage sensitivity calculation are based on the updated Jacobian matrix, which can only calculate coefficients related to the power injections. Since the voltage sensitivity with respect to the tap changers is needed for the proposed control scheme, the analytical sensitivity calculation method in [33] is used in this paper. Define $S$ and $\Gamma$ as the set of slack buses and the buses with power injection. $\bar{V}_{i}=V_{i} e^{j \theta_{i}}$ denotes the voltage of bus $i$ for $i \in S \cup \Gamma$. The voltage sensitivity with respect to power injections can be calculated by,

$$
\begin{aligned}
& \frac{\partial V_{i}}{\partial P_{l}}=\frac{1}{V_{i}} \operatorname{Re}\left(\underline{V_{i}} \frac{\partial \bar{V}_{i}}{\partial P_{l}}\right) \\
& \frac{\partial V_{i}}{\partial Q_{l}}=\frac{1}{V_{i}} \operatorname{Re}\left(V_{i} \frac{\partial \bar{V}_{i}}{\partial Q_{l}}\right)
\end{aligned}
$$

where $P_{l}$ and $Q_{l}$ denote the active and reactive power injection of bus $l-(l \in \Gamma)$, respectively; $\underline{V_{i}}$ denote the conjugate of $\bar{V}_{i} ; \partial \bar{V}_{i} / \partial P_{l}$ and $\partial \bar{V}_{i} / \partial Q_{l}$ can be derived from $\bar{V}_{i}$ and $\bar{Y}_{b u s, i j}\left(\bar{Y}_{b u s, i j}\right.$ denotes the elements of the admittance matrix $\left.\left[\bar{Y}_{\text {bus }}\right]_{N \times N}\right)$; and $\operatorname{Re}($ ) represents the real part of the complex term in the parentheses.

The voltage sensitivity coefficients with respect to tap changes is difficult to calculate directly. Considering the relationship between tap changes and the voltage of MV bus ( $V_{k}$ ), the voltage sensitivity with respect to the MV bus 
voltage can be calculated firstly and then the voltage sensitivity with respect to tap changes can be derived by,

$$
\frac{\Delta V_{i}}{\Delta N_{\text {tap }}}=V_{k 1} \cdot \Delta V_{\text {tap }} \cdot \frac{V_{N 1}}{V_{N 2}} \cdot \frac{\partial V_{i}}{\partial V_{k}}
$$

where $V_{k 1}$ is the primary voltage of the transformer and $\Delta V_{\text {tap }}$ is the voltage step per tap; $V_{N 1}$ and $V_{N 2}$ denote the nominal voltage of the transformer; and $\partial V_{i} / \partial V_{k}$ is the sensitivity coefficients with respect to the MV bus voltage magnitude, which can be calculated directly.

2) Critical bus selection:

To minimize the voltage deviation from the nominal value within distribution networks, the real-time operation states of the network need to be monitored. However, considering the heavy burden of investment and computation, it is impractical to monitor all bus voltages in the network. Therefore, several critical buses should be selected to reflect the whole system operation conditions. In this paper, a systematic critical bus selection method based on clustering techniques is used. The electrical distance is only related to the sensitivity coefficients $\partial V / \partial Q$, which is suitable for the network with low $R / X$ radio [27]. In distribution networks, the impact of active power injections should be taken into account. Therefore, the degree of coupling between buses $i$ and $j$ is quantified by,

$$
d_{i j}=\frac{\left|\frac{\partial V_{i}}{\partial P_{j}}\right|+\left|\frac{\partial V_{i}}{\partial Q_{j}}\right|}{\left|\frac{\partial V_{j}}{\partial P_{j}}\right|+\left|\frac{\partial V_{j}}{\partial Q_{j}}\right|}
$$

then, the electrical distance between nodes $i$ and $j$ is defined as,

$$
D_{i j}=-\log \left(d_{i j} \cdot d_{j i}\right)
$$

Based on the electrical distance, the hierarchical clustering method is used to group buses [36]. Initially, each bus represents a cluster, and then the clusters gradually merge to the pre-determined threshold. After that, the critical buses of each group can be selected by the following principle: the DGconnected buses will be selected as the critical buses; otherwise, the buses at the end of feeder are selected. In this paper, DG-connected buses are selected as the critical buses and the results of the critical bus selection are showed in Fig.4 (critical buses in red). The non-critical bus voltages are assumed to be close to the critical bus voltages due to the small electrical distance.

\section{MPC-Based Coordinated Voltage Control}

Model predictive control is a widely used optimal control method. The control input is obtained by solving a discretetime optimal control problem over a given horizon. An optimal control input sequence is generated and only the first control in the sequence is applied [37].

The principle of MPC is illustrated in Fig. 2. In a prediction horizon $T_{P}$, there are $N_{C}$ control periods $T_{C}$.For OLTC control, $T_{C}$ is set in seconds and must be equal or longer than the mechanical time delay $T_{\text {mech }}$, which is much longer than the response time of DG units. To achieve coordinated voltage control, the sampling time $\Delta T_{P}$ is set as the control time of DG and STATCOM. The number of prediction steps within one control period and total prediction steps are $N_{S}=T_{C} / \Delta T_{P}$ and $N_{P}=T_{P} / \Delta T_{P}$, respectively.

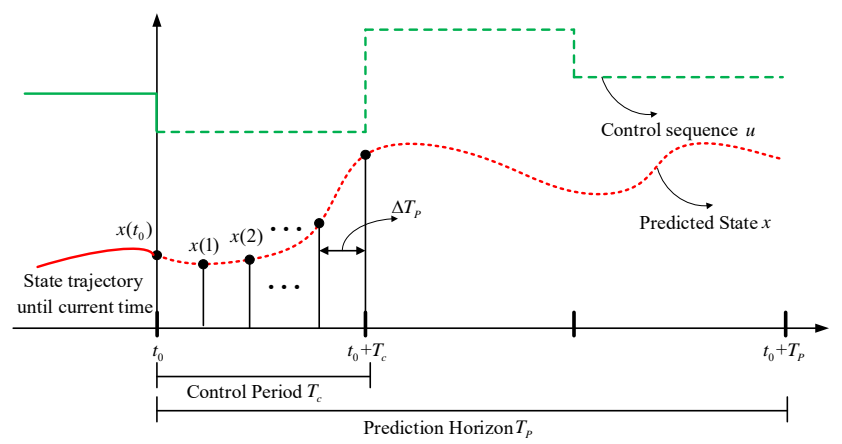

Fig. 2. Principle of MPC.

1) Objective function: Firstly, the OLTC controller minimizes the voltage deviation from the nominal value within distribution networks. Secondly, reducing the operation times of the OLTC is also be considered. Accordingly, the cost function is expressed as,

$$
\min _{\mathbf{n}_{\text {tap }}} \sum_{i=1}^{N_{C}} \sum_{k=1}^{N_{S}}\left(\left\|\mathbf{V}_{i}(k)-\mathbf{V}_{\text {ref }}\right\|_{W_{v}}^{2}+\left\|\mathbf{n}_{\text {tap }}(i)-\mathbf{n}_{\text {tap }}(i-1)\right\|_{\text {Wtap }}^{2}\right)
$$

where $\mathbf{V}_{\text {ref }}$ denotes the voltage reference, which is typically 1.0 p.u. and $\mathbf{V}_{i}(k)$ is the vector of the predictive voltage, $\mathbf{n}_{\text {tap }}$ is the tap position of the OLTC, $W_{v}$ and $W_{\text {tap }}$ are the weighting factors for minimizing the variation of voltage and variation of tap position, respectively.

2) Constraints: For an OLTC transformer, the allowable operation range of the tap is as follows,

$$
\begin{gathered}
\underline{n}_{\text {tap }} \leq n_{\text {tap }}(i) \leq \bar{n}_{\text {tap }}, \forall i \\
\Delta \underline{n}_{\text {tap }} \leq \Delta n_{\text {tap }}(i) \leq \Delta \bar{n}_{\text {tap }}, \forall i
\end{gathered}
$$

where $\underline{n}_{\text {tap }}$ and $\bar{n}_{\text {tap }}$ are the lower and upper limits of the tap position, $\Delta \underline{n}_{\text {tap }}$ and $\Delta \bar{n}_{\text {tap }}$ are the lower and upper limits of the tap changes. The predictive voltage derived from (24) is,

$$
\mathbf{V}_{i}(k)=\mathbf{V}(0)+\frac{\partial V}{\partial N_{\text {tap }}} \Delta n_{\text {tap }}(i)+\frac{\partial V}{\partial Q^{\mathrm{T}}} \Delta \mathbf{Q}(k)+\frac{\partial V}{\partial P_{D G}^{\mathrm{T}}} \Delta \overline{\mathbf{P}}_{D G}(k), \forall k
$$

where $\mathbf{P}_{D G}$ is the forecasted available power of DG units, which can be obtained hours-ahead according to the meteorological data, $\mathbf{V}(0)$ denote the measured voltage magnitude at the current operating point, $\Delta \overline{\mathbf{P}}_{D G}$ and $\Delta \mathbf{Q}(k)$ denote the variations of active/reactive power injections, respectively. $\mathbf{V}(0)$ and $\Delta \mathbf{Q}(k)$ are provided by local controllers.

The presented MPC problem can be reformulated as a standard Integer Quadratic Programming (IQP) problem with the decision variable $\Delta n_{\text {tap }}(i)$. Generally, the control actions are only changed at the beginning of the control period and maintained within the control period. The optimal action command is calculated based on the transient state, which may cause OLTC mis-operation. In order to avoid OLTC misoperation, the OLTC will receive the command and perform a 
tap change only if the optimal action command is maintained for a predefined time delay $T_{\text {delay }}$.

\section{Flowchart and Architecture of the DCVC Scheme}

Fig. 3 shows the flowchart of the whole control strategy to better illustrate the relationship between controllers. The steps of the distributed coordinated voltage control method can be described as follows:

- Local controller calculates the optimal reactive power outputs of DG units and STATCOM according to the local voltage and current reactive power injection based on the GP method;

- Local controllers send the measured voltage and calculated reactive power outputs to the OLTC controller; and

- OLTC controller calculates the optimal operation command based on the information received and perform a tap change after a time delay.

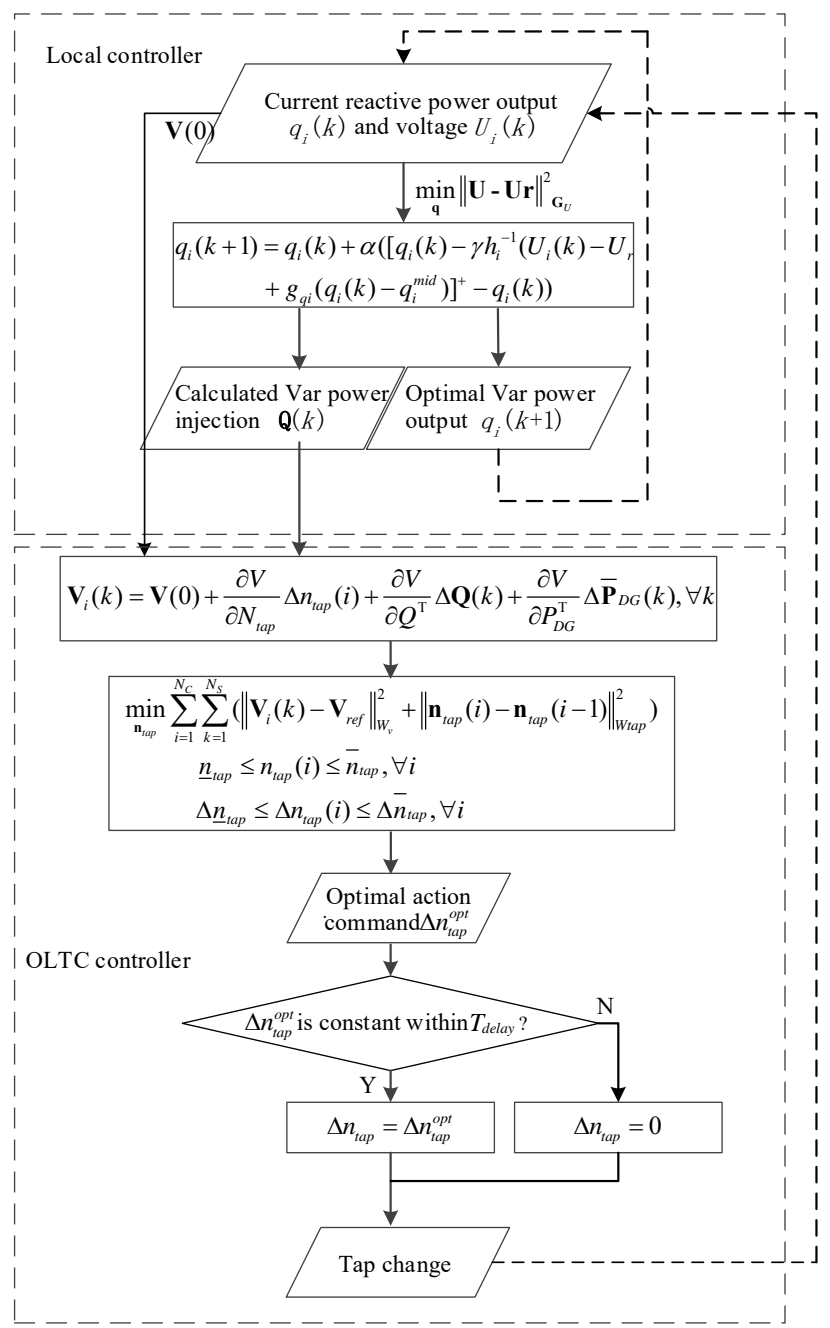

Fig. 3. Flowchart of the proposed distributed coordinated voltage control.

The distributed coordinated voltage control architecture is shown in Fig. 4 to better illustrate the unidirectional communication based coordination mechanism. Each DG and STATCOM is equipped with a smart agent which can send information and solve the control problems independently.
The measured voltages and reactive power outputs of DG units and STATCOM are sent from the local controller to the OLTC controller using one-way communication. The tap change of the OLTC affects the optimization calculation in the local controller by changing the voltages within the distribution network. Therefore, a unidirectional communication based coordination mechanism between the fast var devices and OLTC is achieved. According to [38], the minimum information update interval in IEEE 802.11 (Wi-Fi) is of the order of $10 \mathrm{~ms}$, which is adequate for the proposed DCVC system. Therefore, It is possible to apply the proposed control strategy in a real distribution network.

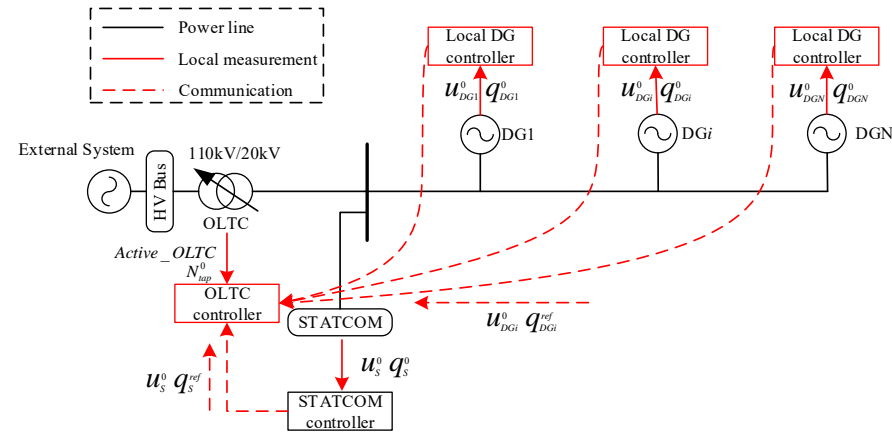

Fig. 4. Architecture of proposed distributed coordinated voltage control.

\section{CASE Study}

In this section, the performance of the proposed distributed coordinated voltage control scheme is tested on a Finnish distribution network equipped with a \pm 1 MVar STATCOM. The network topology is shown in Fig. 5. Two $20 \mathrm{kV}$ feeders are connected to the same medium voltage (MV) bus equipped with a STATCOM. Eight DG units are connected to these feeders. More details of the ADN parameters can be found in [39]. In addition, the external system supplies power to the distribution network.

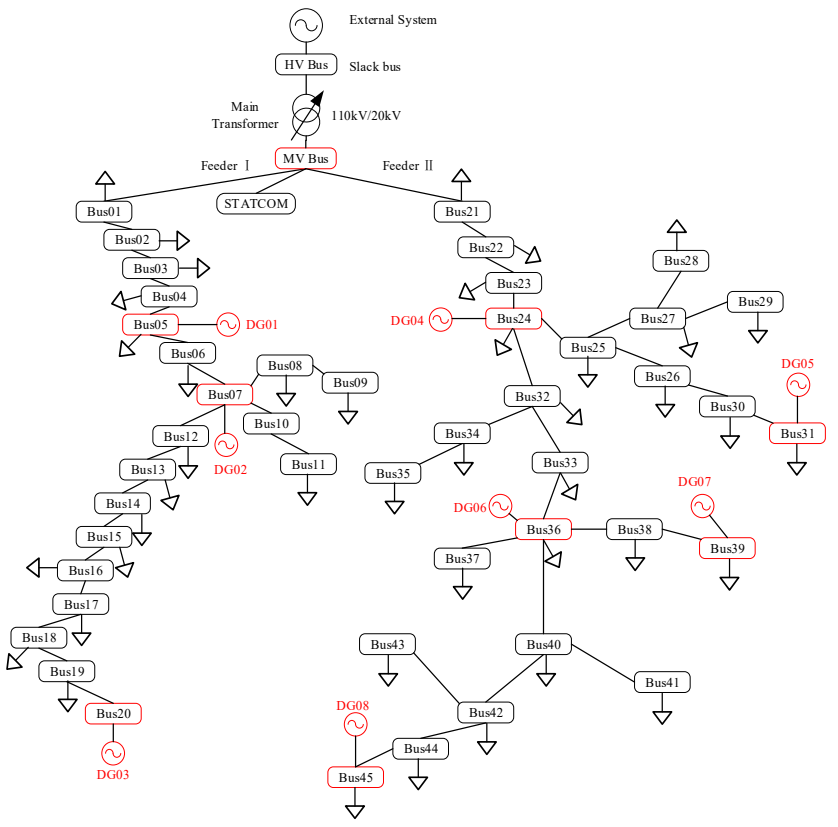

Fig. 5. Structure of an active distribution network. 
The DG and STATCOM control is carried out every $0.5 \mathrm{~s}$, $\alpha$ is set as 0.9 and $\gamma$ is set as $0.8 * 2 / \lambda_{\max }\left(\mathbf{H}^{-1} \mathbf{M}\right)$. The tap changing range of the OLTC is $\pm 9 \times 1.67 \%$ and the mechanical time delay is $5 \mathrm{~s}$. The control period $T_{C}$, prediction horizon $T_{P}$ and the sampling time $\Delta T_{P}$ of the MPC are designed as $5 \mathrm{~s}, 15 \mathrm{~s}, 0.5 \mathrm{~s}$, respectively (i.e., $N_{S}=10$, $N_{C}=3$ ). The sampling computation period of the OLTC controller $\bar{T}_{C}=\Delta T_{P}=0.5 \mathrm{~s}$ and the predefined time to prevent OLTC mis-operation $T_{\text {delay }}$ is set as $3 \mathrm{~s}$. Firstly, for the decentralized reactive power control of DG and STATCOM, the results concerning the convergence performance with different control parameters are given. Secondly, the control performance under different operating conditions are tested.

\section{A. Convergence Performance}

The convergence performance of the proposed reactive power control of DG and STATCOM with different value of the control parameters is presented in this subsection. The iteration number is set as 30 . When testing the convergence performance with different $\gamma, \alpha$ is set as 0.9 . When testing the convergence performance with different $\alpha, \gamma$ is set as $0.8^{*} 2 / \lambda_{\max }\left(\mathbf{H}^{-1} \mathbf{M}\right)$.
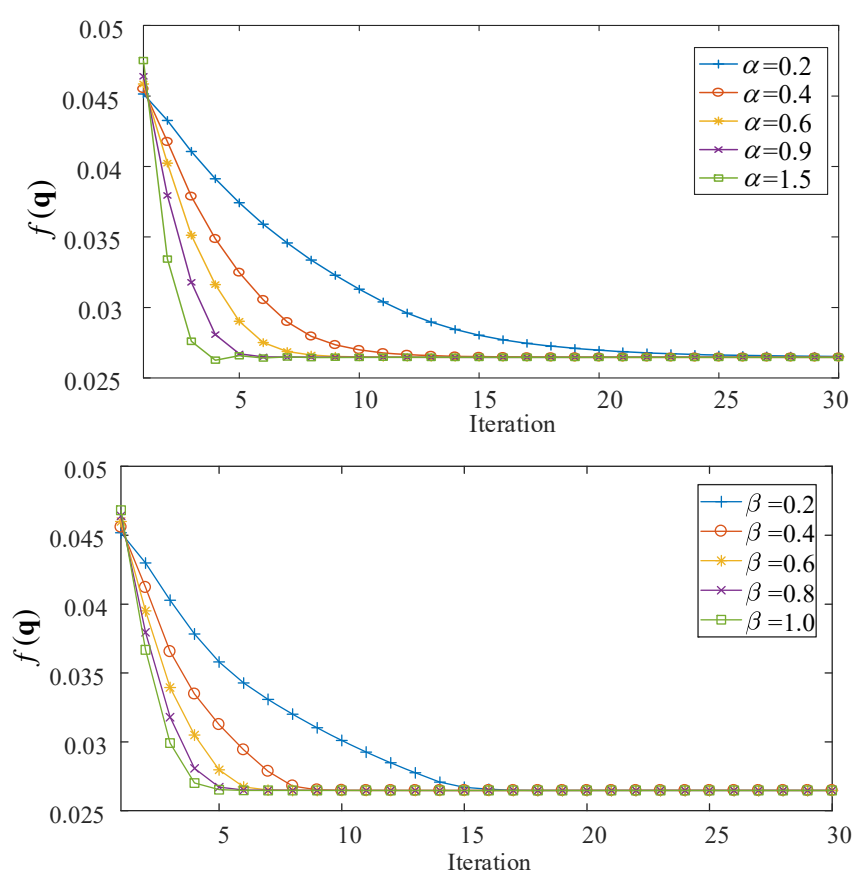

Fig. 6. Convergence performance of decentralized voltage control with different $\alpha$ and $\beta$.

Fig. 6 shows the convergence performance with different $\alpha$ and $\beta$, where the parameter $\beta$ is defined as $\gamma=\beta^{*} 2 / \lambda_{\max }\left(\mathbf{H}^{-1} \mathbf{M}\right)$. It can be seen that the value of $\alpha$ and $\gamma$ are closely related to the convergence and stability of the proposed algorithm. The control scheme is more stable with small $\alpha$ and $\gamma$, and shows much better convergence when $\alpha$ and $\gamma$ are larger.

In order to achieve faster convergence and more stable results in various scenarios, $\alpha$ is set as 0.9 and $\gamma$ is set as $0.8 * 2 / \lambda_{\max }\left(\mathbf{H}^{-1} \mathbf{M}\right)$ in this paper.

The convergence performance of the scaled GP method and basic GP method is shown in Fig. 7. It can be seen that the scaled GP method converges to the optimal solution faster than the basic one.

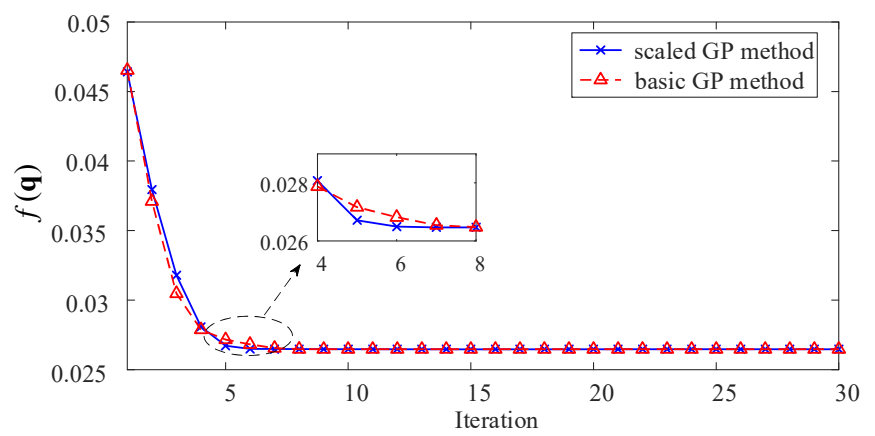

Fig. 7. Convergence performance of basic GP method and scaled GP method.

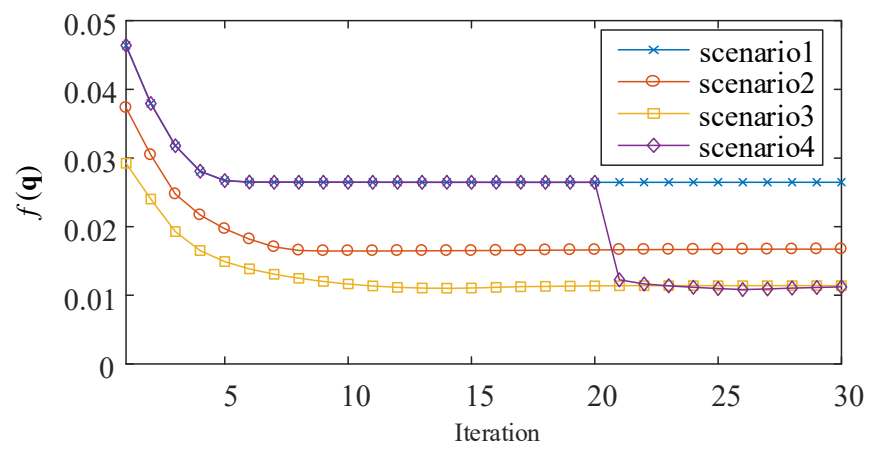

Fig. 8. Convergence performance of the decentralized algorithm under different scenarios.

The convergence performance of the decentralized algorithm under different scenarios is shown in Fig. 8. Four scenarios are simulated according to various DG outputs: Scenario1: $\quad P_{D G}=0.9 S_{D G} \quad, \quad$ Scenario2: $\quad P_{D G}=0.8 S_{D G}$, Scenario3: $P_{D G}=0.7 S_{D G}$ and Scenario4: $P_{D G}$ changes from $0.9 S_{D G}$ to $0.7 S_{D G}$. It can be seen that the decentralized algorithm can converge stably under different scenarios.

\section{B. Voltage Control Performance}

In this subsection, the control performance of the proposed DCVC method is examined and compared with the ones based on the centralized model predictive control (CMPC), the ones with the LDC control for the OLTC and the ones without control of DG reactive power outputs (NC). For the centralized MPC control, the central controller obtains the global information and calculates the optimal reactive power support of the DG and STATCOM based on the MPC method. The total simulation time is set as $120 \mathrm{~s}$ and the control period of the CMPC control is set as $2 \mathrm{~s}$.

\section{1) Normal Operation}

The control performance under normal operation only considering the fluctuations of DG power outputs and network load is tested in this subsection. Fig. 9 shows the mean voltage deviation of all critical buses, which reflects the overall voltage control performance of the distribution network. It can be seen that the DCVC and CMPC can both effectively regulate the voltages with small deviations and fluctuations 
caused by the active power variations of DG units. Moreover, the voltage control performance of the DCVC is close to that of the CMPC.

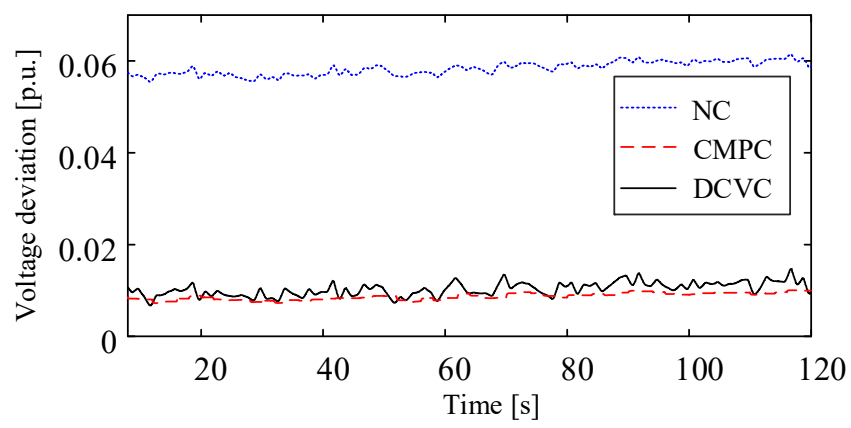

Fig. 9. Mean voltage deviation of all buses.

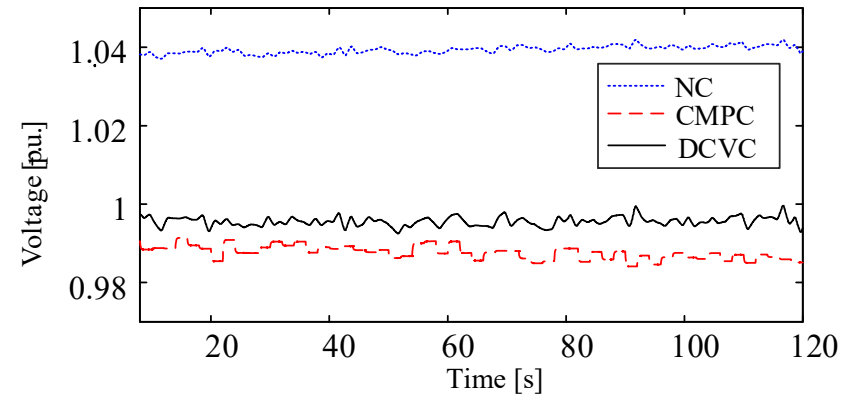

Fig. 10. Voltage of Bus05.

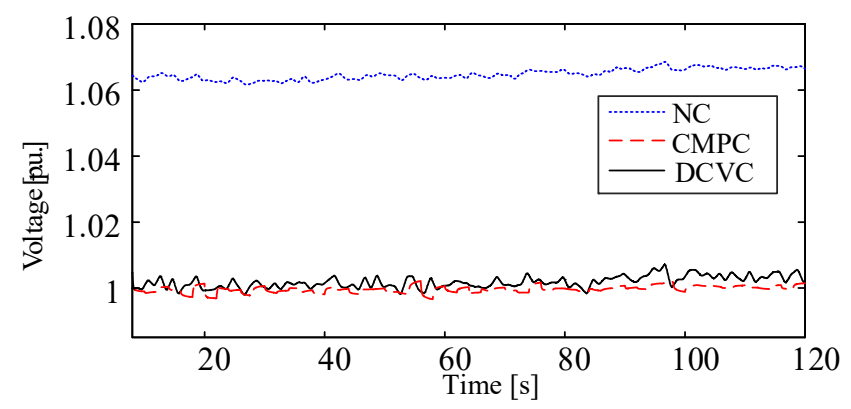

Fig. 11. Voltage of Bus45.

Figs. 10 and 11 show the voltage of Bus 05 connected with DG01 and Bus 45 at the end of feeder. Both the DCVC and CMPC schemes can improve the voltage performance and the voltage control performance of the DCVC control is close to that of the centralized CMPC control. With the proposed DCVC scheme, the voltages of Bus 05 are better than the ones with the CMPC method.

2) Under Disturbance

In this subsection, to illustrate the control performance under a large disturbance, the distribution network is simulated under the influence of large disturbances from the external network. There is a sudden voltage decrease of 0.1 p.u. at the slack bus $(t=50 \mathrm{~s})$, which causes all bus voltages decrease in the network. Fig. 12 shows the mean voltage deviation of all buses. As can be seen, the proposed DCVC strategy ,CMPC and LDC can better regulate the voltages and voltage deviations are smaller. Fig. 13 shows the tap position changes of the OLTC. It can be seen that the proposed DCVC scheme effectively reduces the operation times of the OLTC, while the CMPC and LDC have more OLTC operation times. The difference is that the OLTC adopts automatic voltage control according to the voltage of the MV Bus in the CMPC and LDC scheme. However, the proposed DCVC scheme minimizes the operations times of the OLTC while minimizing the voltage deviation from the nominal value within distribution networks.

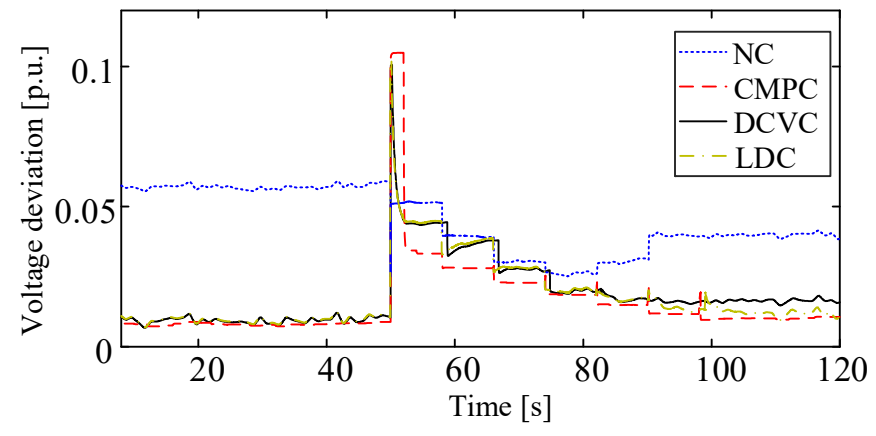

Fig. 12. Mean voltage deviation of all buses.

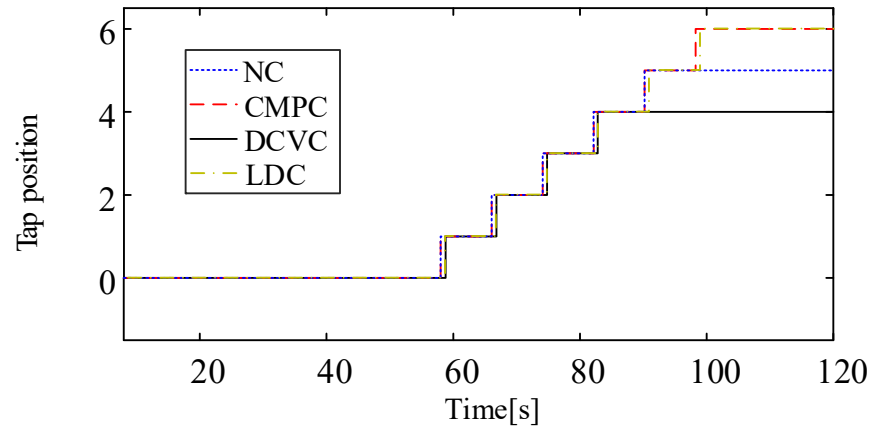

Fig. 13. Tap position change of OLTC.

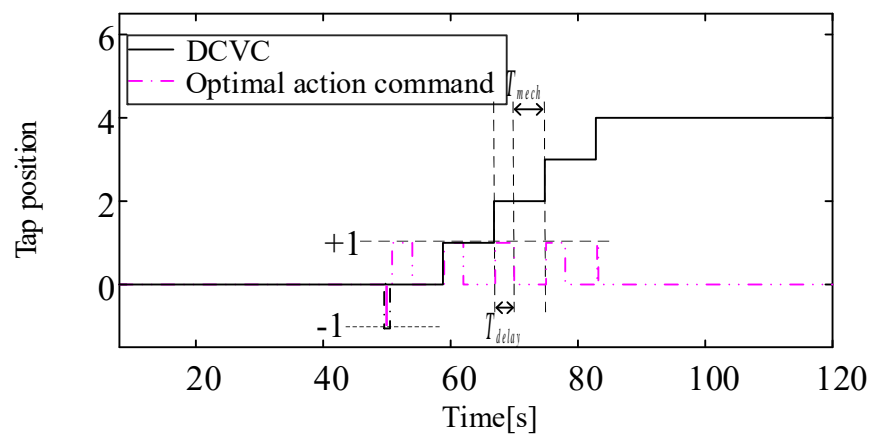

Fig. 14. Control signal delay of OLTC.

The relationship between the optimal action command calculation and tap position changes is showed in Fig. 14. It can be seen that the OLTC transformer will receive the command and then act after the mechanical time delay $T_{m e c h}$ only when the duration of the optimal action command exceeds the predefined time $T_{\text {delay }}$. In this way, the unnecessary operation of the OLTC is reduced.

3) Long-Time-Scale

To verify the control performance of the proposed DCVC scheme in a longer time-scale, the total simulation time is set as $1000 \mathrm{~s}$ in this subsection. Fig. 15 shows the mean voltage deviation of all buses. It can be seen that the proposed DCVC 
can effectively regulate the voltages with small deviations in a long time-scale.

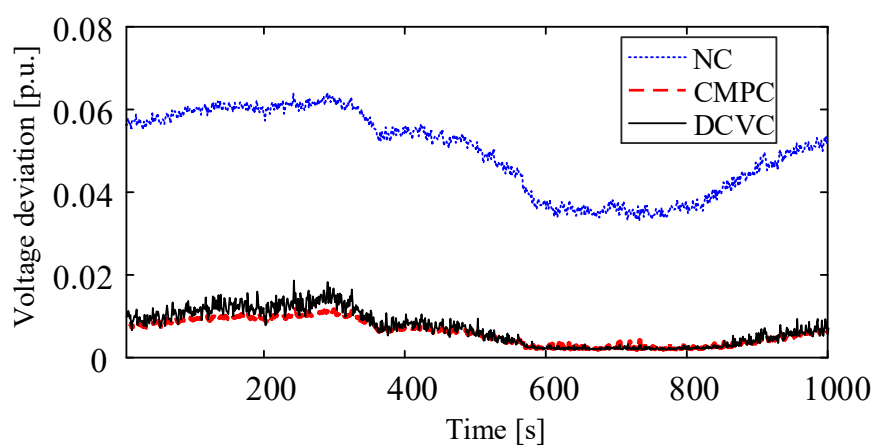

Fig. 15. Mean voltage deviation of all buses.

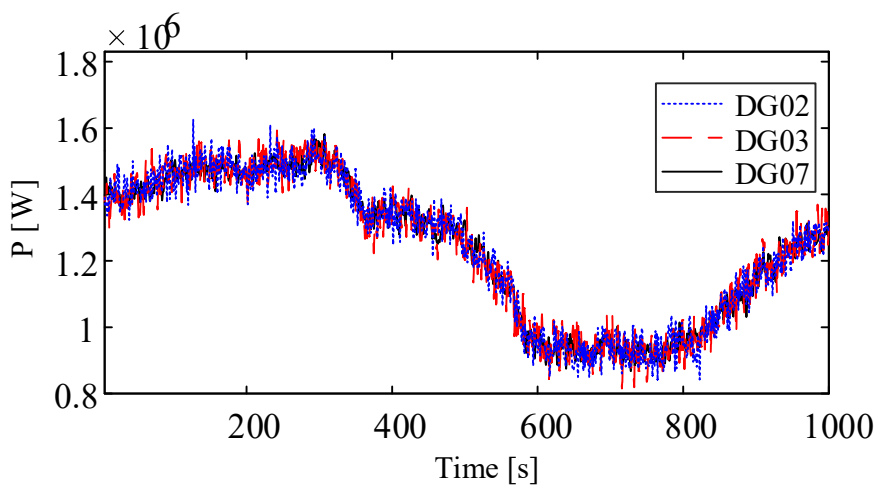

Fig. 16. Active power output of DG units.

The variations of the DG outputs are shown in Fig. 16 and the reactive power outputs of DG units and STATCOM optimized in the proposed DCVC scheme are shown in Fig. 17. It can be seen that the reactive power outputs of DG units and STATCOM are adjusted with the voltage fluctuation caused by the variations of the DG outputs.

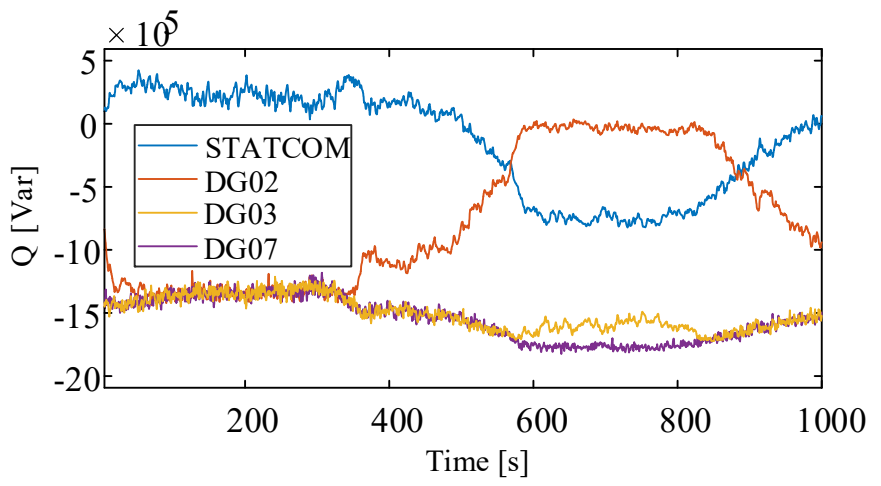

Fig. 17. Reactive power outputs of DG units.

\section{CONCLUSION}

In this paper, a distributed coordinated voltage control scheme based on MPC and the GP method is proposed for distribution networks with DG units and OLTC. The control scheme considers voltage regulation devices with different temporal characteristics and their coordination: DG and STATCOM adopt decentralized control to calculate the optimal reactive power outputs locally and coordinate with the
OLTC; the OLTC controller adopts the model predictive control (MPC) to calculate the optimal action command and coordinates with DG units through unidirectional communication. As such, it meets the requirement of fast voltage adjustment and ensures less switching operations of the OLTC with slow dynamics. Moreover, it also reduces the calculation burden of voltage controllers in the distribution network. As verified by the case studies, the coordinated voltage control scheme can efficiently maintain the voltages within the distribution network close to the nominal value and smaller fluctuations under the normal condition and reduce the operation times of the OLTC under the large-disturbance condition.

\section{APPENDIX}

\section{OPTIMALITY AND STABILITY ANALYSIS}

\section{A. Optimality Analysis}

The weight matrix $\mathbf{G}_{U}$, as mentioned in Section III, is positive definite; the reactive power constraint set $\left[\mathbf{q}^{\min }, \mathbf{q}^{\max }\right.$ ] is a closed loop convex set; and the objective function $f(\mathbf{q})$ is obviously convex. Therefore, for a given $\overline{\mathbf{U}}$, the sufficient condition under which $\mathbf{q}^{*}$ is the global optimal solution is,

$$
\nabla f\left(\mathbf{q}^{*}\right)^{\mathrm{T}}\left(\mathbf{q}-\mathbf{q}^{*}\right) \geq 0, \forall \mathbf{q} \in\left[\mathbf{q}^{\min }, \mathbf{q}^{\max }\right]
$$

by multiplying both sides with $-\gamma \mathbf{H}^{-1}$,

$$
\begin{gathered}
\left(\left(\mathbf{q}^{*}-\gamma \mathbf{H}^{-1} \nabla f\left(\mathbf{q}^{*}\right)\right)-\mathbf{q}^{*}\right)^{\mathrm{T}}\left(\mathbf{q}-\mathbf{q}^{*}\right) \leq 0, \\
\forall \mathbf{q} \in\left[\mathbf{q}^{\min }, \mathbf{q}^{\max }\right], \quad \mathbf{H} \succ 0, \quad \gamma>0
\end{gathered}
$$

thus, for a given $\mathbf{q} \in\left[\mathbf{q}^{\min }, \mathbf{q}^{\max }\right]$,

$$
\begin{aligned}
\left\|\mathbf{q}-\left(\mathbf{q}^{*}-\gamma \mathbf{H}^{-1} \nabla f\left(\mathbf{q}^{*}\right)\right)\right\|^{2} & =\left\|\mathbf{q}^{*}-\left(\mathbf{q}^{*}-\gamma \mathbf{H}^{-1} \nabla f\left(\mathbf{q}^{*}\right)\right)\right\|^{2}+\left\|\mathbf{q}-\mathbf{q}^{*}\right\|^{2} \\
& -2\left(\left(\mathbf{q}^{*}-\gamma \mathbf{H}^{-1} \nabla f\left(\mathbf{q}^{*}\right)\right)-\mathbf{q}^{*}\right)^{\mathrm{T}}\left(\mathbf{q}-\mathbf{q}^{*}\right) \\
& \geq\left\|\mathbf{q}^{*}-\left(\mathbf{q}^{*}-\gamma \mathbf{H}^{-1} \nabla f\left(\mathbf{q}^{*}\right)\right)\right\|^{2}
\end{aligned}
$$

Formula (38) means that the optimal solution $\mathbf{q}^{*}$ is equivalent to the value closest to the vector $\left(\mathbf{q}^{*}-\gamma \mathbf{H}^{-1} \nabla f\left(\mathbf{q}^{*}\right)\right)$ in the constraint set $\left[\mathbf{q}^{\min }, \mathbf{q}^{\max }\right]$, i.e.,

$$
\mathbf{q}^{*}=\left[\left(\mathbf{q}^{*}-\gamma \mathbf{H}^{-1} \nabla f\left(\mathbf{q}^{*}\right)\right)\right]^{+}
$$

The optimal solution in (39) is exactly the result that the iteration in (18) converges to. Thus, if the decentralized control method converges to a solution, it must be the optimal within the constraint set.

\section{B. Stability Analysis}

The range of control parameter that enables the iteration to converge is calculated in this part. According to (18), it can be obtained that,

$$
\begin{aligned}
\left\|\mathbf{q}(k+1)-\mathbf{q}^{*}\right\|= & \|(1-\alpha)\left(\mathbf{q}(k)-\mathbf{q}^{*}\right) \\
& +\alpha\left[\mathbf{q}(k)-\gamma \mathbf{H}^{-1} \nabla f(\mathbf{q}(k))\right]^{+}-\alpha \mathbf{q}^{*} \| \\
\leq & (1-\alpha)\left\|\mathbf{q}(k)-\mathbf{q}^{*}\right\| \\
& +\alpha\left\|\left[\mathbf{q}(k)-\gamma \mathbf{H}^{-1} \nabla f(\mathbf{q}(k))\right]^{+}-\mathbf{q}^{*}\right\|
\end{aligned}
$$

According to the mapping method (12) and equation (39), 


$$
\begin{aligned}
\left\|\mathbf{q}(k+1)-\mathbf{q}^{*}\right\| \leq & \alpha \|\left[\mathbf{q}(k)-\gamma \mathbf{H}^{-1} \nabla f(\mathbf{q}(k))\right]^{+} \\
& -\left[\left(\mathbf{q}^{*}-\gamma \mathbf{H}^{-1} \nabla f\left(\mathbf{q}^{*}\right)\right)\right]^{+}\|+(1-\alpha)\| \mathbf{q}(k)-\mathbf{q}^{*} \| \\
\leq & \alpha \| \mathbf{q}(k)-\mathbf{q}^{*}-\gamma \mathbf{H}^{-1}\left(\nabla f\left(\mathbf{q}(k)-\nabla f\left(\mathbf{q}^{*}\right)\right) \|\right. \\
& +(1-\alpha)\left\|\mathbf{q}(k)-\mathbf{q}^{*}\right\|
\end{aligned}
$$

then substitute the gradient $\nabla f(\mathbf{q}(k))=\mathbf{M q}(\mathbf{k})+\overline{\mathbf{U}}-\mathbf{U}_{\mathrm{r}}$ into the right side of the inequality,

$$
\begin{aligned}
\left\|\mathbf{q}(k+1)-\mathbf{q}^{*}\right\| & \leq(1-\alpha)\left\|\mathbf{q}(k)-\mathbf{q}^{*}\right\|+\alpha\left\|\left(\mathbf{I}-\gamma \mathbf{H}^{-1} \mathbf{M}\right)\left(\mathbf{q}(k)-\mathbf{q}^{*}\right)\right\| \\
& \leq\left(1-\alpha+\alpha\left\|\mathbf{I}-\gamma \mathbf{H}^{-1} \mathbf{M}\right\|\right)\left\|\mathbf{q}(k)-\mathbf{q}^{*}\right\|
\end{aligned}
$$

Thus, a sufficient stability condition is that $\mathbf{q}(k+1)$ is closer to $\mathbf{q}^{*}$ than $\mathbf{q}(k)$, and this is equivalent to $\left\|\mathbf{I}-\gamma \mathbf{H}^{-1} \mathbf{M}\right\|<1$.

Define the matrix $\mathbf{D}:=\mathbf{H}^{1 / 2}\left(\mathbf{H}^{-1} \mathbf{M}\right) \mathbf{H}^{-1 / 2}=\mathbf{H}^{-1 / 2} \mathbf{M} \mathbf{H}^{-1 / 2}$ and for any given n-dimensional vector $\mathbf{x}$,

$$
\mathbf{x}^{\mathrm{T}} \mathbf{D} \mathbf{x}=\left(\mathbf{H}^{-1 / 2} \mathbf{x}\right)^{\mathrm{T}} \mathbf{M}\left(\mathbf{H}^{-1 / 2} \mathbf{x}\right)>0
$$

obviously, matrix $\mathbf{D}$ and $\mathbf{H}^{-1} \mathbf{M}$ are both positive definite.

According to the principle that the matrix Euclidean norm equals to its largest singular value, $\left\|\mathbf{I}-\gamma \mathbf{H}^{-1} \mathbf{M}\right\|<1$ holds if,

$$
\left|1-\gamma \lambda_{i}\left(\mathbf{H}^{-1} \mathbf{M}\right)\right|_{\max }<1
$$

where $\lambda_{i}\left(\mathbf{H}^{-1} \mathbf{M}\right)>0$ denotes an eigenvalue of the matrix. Besides, due to $\gamma>0$, the sufficient condition for iteration convergence is,

$$
\gamma<\frac{2}{\lambda_{\max } \mathbf{H}^{-1} \mathbf{M}}
$$

\section{REFERENCES}

[1] A. Mohamed, and T. J. T. Hashim, "Coordinated voltage control in active distribution networks," in Electric Distribution Network Management and Control, 2018, pp. 85-109.

[2] P. Hallberg, et al. "Active Distribution System Management-A Key Tool For The Smooth Integration Of Distributed Generation," Eurelectric, Brussels, Belgium, Feb. 2013.

[3] N. Mahmud, A. Zahedi, "Review of control strategies for voltage regulation of the smart distribution network with high penetration of renewable distributed generation," Renew. Sustain. Energy Rev., vol. 64, pp. 582-595, 2016.

[4] Y. P. Agalgaonkar, B. C. Pal, and R. A. Jabr, "Distribution voltage control considering the impact of PV generation on tap changers and autonomous regulators," IEEE Trans. Power Syst., vol. 29, no. 1, pp. 182-192, Jan. 2014.

[5] Y. Guo, Q. Wu, H. Gao, X. Chen, J. Østergaard, and H. Xin, "MPCBased Coordinated Voltage Regulation for Distribution Networks With Distributed Generation and Energy Storage System," IEEE Trans. Sustain. Energy, vol. 10, no. 4, pp.1731-1739, Oct. 2019.

[6] A. D. T. Le, K. Muttaqi, M. Negnevitsky, and G. Ledwich, "Applying Protection Principles for Controlling Distributed Generation," Australian Journal of Electrical and Electronics Engineering, vol. 4, no. 3, pp.249254, Jan. 2008.

[7] P. N. Vovos, A. E. Kiprakis, and A. R. Wallace, "Centralized and distributed voltage control: impact on distributed generation penetration," IEEE Trans. Power Syst., vol. 22 no. 1, pp. 476-483, Feb. 2007.

[8] V. Calderaro, G. Conio, V. Galdi, G. Massa, and A. Piccolo, "Optimal decentralized voltage control for distribution systems with inverterbased distributed generators," IEEE Trans. Power Syst., vol. 29, no. 1, pp. 230- 241, Jan. 2014.
[9] G. Cavraro, and R. Carli, "Local and distributed voltage control algorithms in distribution network," IEEE Trans. Power Syst., vol. 33, no. 2, pp.1420-1430, Mar. 2018.

[10] C. Gao, and M. A. Redfern, "A review of voltage control techniques of networks with distributed generations using on-load tap changer transformers," in Proc. $45^{\text {th }}$ Int. Universities Power Eng. Conf., October 2010, pp. 1-6.

[11] Q. Zhou, and J. W. Bialek, "Generation curtailment to manage voltage constraints in distribution networks," IET Gener. Transm. Distrib., vol. 1, pp. 4929-498, 2007.

[12] S. Weckx, C. Gonzalez, and J. Driesen, "Combined central and local active and reactive power control of PV inverters," IEEE Trans. Sustain. Energy, vol. 5, no. 3, pp. 776-784, Jul. 2014.

[13] L. A. Kojovic, "Coordination of distributed generation and step voltage regulator operations for improved distribution system voltage regulation," in Proc. IEEE Power Eng. Soc. Gen. Meet., January 2006, pp. 1-4.

[14] Y. J. Kim, J. L. Kirtley, and L, K, Norford, "Reactive power ancillary service of synchronous DGs in coordination with voltage control devices," IEEE Trans. Smart Grid, vol. 8, no. 2, pp. 515-527, Mar. 2017.

[15] K. Christakou, D. C. Tomozei, J. L. Boudec, and M. Paolone, "GECN: Primary voltage control for active distribution networks via real-time demand-response," IEEE Trans. Smart Grid, vol. 5, no. 2, pp. 622-631. Mar. 2014.

[16] V. Zamani, A. Cortes, and J. Kleissl, "Integration of PV generation and storage on power distribution systems using MPC," in Proc. IEEE Power Energy Soc. Gen. Meet., July 2015, pp. 1-5.

[17] G. Cavraro, T. Caldognetto, and R. Carli, "A master/slave control of distributed energy resources in low-voltage microgrids," in Proc. 2016 European Control Conf., Aalborg, June 2016, pp. 1507-1512.

[18] H. S. Bidgoli, and T. V. Cutsem, "Combined Local and Centralized Voltage Control in Active Distribution Networks," IEEE Trans. Power Syst., vol. 33 no.pp.1374-1384, Mar. 2018.

[19] P. Li, J. Ji, H. Ji, J. Jian, F. Ding, J. Wu, and C. Wang, "MPC-Based Local Voltage Control Strategy of DGs in Active Distribution Networks," IEEE Trans. Sustain. Energy, vol. 11, no. 4, pp. 2911-2921, Oct. 2020 .

[20] S. Maharjan, A. M. Khambadkone, and J. C. -H. Peng, "Robust Constrained Model Predictive Voltage Control in Active Distribution Networks," IEEE Trans. Sustain. Energy, vol. 12, no. 1, pp. 400-411, Jan. 2021.

[21] L. Cheng, Y. Chang, and R. Huang, "Mitigating voltage problem in distribution system with distributed solar generation using electric vehicles," IEEE Trans. Sustain. Energy, vol. 6, no. 4, pp. 1475-1484, Oct. 2015.

[22] M. E. Baran, and I. M. El-Markabi, "A Multiagent-Based Dispatching Scheme for Distributed Generators for Voltage Support on Distribution Feeders," IEEE Trans. Power Syst., vol. 22, no. 1, pp. 52-59, Feb. 2007.

[23] P. Sulc, S. Backhaus, and M. Chertkov, "Optimal Distributed Control of Reactive Power Via the Alternating Direction Method of Multipliers," IEEE Trans. Energy Convers., vol. 29, no. 4, pp. 968-977, Aug. 2014.

[24] G. Lou, W. Gu, Y. Xu, M. Cheng, and W. Liu, "Distributed MPC-based secondary voltage control scheme for autonomous droop-controlled microgrids," IEEE Trans. Sustain. Energy, vol. 8, no. 2, pp. 792-804, Apr. 2017.

[25] J. Lai, H. Zhou, X. Lu, X. Yu, and W. Hu, "Droop-Based Distributed Cooperative Control for Microgrids with Time-Varying Delays," IEEE Trans. Smart Grid, vol. 7, no. 4, pp. 1775-1789, Jul. 2016.

[26] H. E. Z. Farag, E. F. El-Saadany, and R. Seethapathy, "A Two Ways Communication-Based Distributed Control for Voltage Regulation in Smart Distribution Feeders," IEEE Trans. Smart Grid, vol. 3, no. 1, pp. 271-281, Mar. 2012.

[27] H. E. Z. Farag, and E. F. El-Saadany, "A Novel Cooperative Protocol for Distributed Voltage Control in Active Distribution Systems," IEEE Trans. Power Syst., vol. 28, no. 2, pp. 1645-1656, May 2013.

[28] K. E. Antoniadou-Plytaria, I. N. Kouveliotis-Lysikatos, P. S. Georgilakis, and N. D. Hatziargyriou, "Distributed and decentralized voltage control of smart distribution networks: Models, methods, and future research," IEEE Trans. Smart Grid, vol 8, no. 6, pp. 2999-3008, Nov. 2017.

[29] T. Tewari, A. Mohapatra, and S. Anand, "Coordinated Control of OLTC and Energy Storage for Voltage Regulation in Distribution Network With High PV Penetration," IEEE Trans. Sustain. Energy, vol. 12, no. 1, pp. 262-272, Jan. 2021. 
[30] L. Chen, Z. Deng and X. Xu, "Two-Stage Dynamic Reactive Power Dispatch Strategy in Distribution Network Considering the Reactive Power Regulation of Distributed Generations," IEEE Trans. Power Syst., vol. 34, no. 2, pp. 1021-1032, Mar. 2019.

[31] X. Sun, J. Qiu and J. Zhao, "Real-Time Volt/Var Control in Active Distribution Networks With Data-Driven Partition Method," IEEE Trans. Power Syst., vol. 36, no. 3, pp. 2448-2461, May 2021.

[32] P. Lagonotte, J. C. Sabonnadiere, J. Y. Leost, and J. P. Paul, "Structural analysis of the electrical system: Application to secondary voltage control in France," IEEE Trans. Power Syst., vol. 4, no. 2, pp. 479-486, May 1989.

[33] K. Christakou, J. LeBoudec, M. Paolone, and D. C. Tomozei, "Efficient computation of sensitivity coefficients of node voltages and line currents in unbalanced radial electrical distribution networks," IEEE Trans. Smart Grid, vol. 4, no. 2, pp. 741-750, Jun. 2013.

[34] H. Zhu, and H. Liu, "Fast Local Voltage Control Under Limited Reactive Power: Optimality and Stability Analysis," IEEE Trans. Power Syst., vol. 31, no. 5, pp. 1-10, Sept. 2016.

[35] M. Farivar, L. Chen, and S. Low, "Equilibrium and dynamics of local voltage control in distribution systems," in Proc. IEEE 52nd Conf. Decision and Control (CDC), Dec. 2013, pp. 4329-4334.

[36] H. Sun, Q. Guo, B. Zhang, W. Wu, and B. Wang, "An adaptive zone division-based automatic voltage control system with applications in China," IEEE Trans. Power Syst., vol. 28, no. 2, pp. 1816-1828, May 2013.

[37] E. Camponogara, D. Jia, B. H. Krogh, and S. Talukdar, "Distributed model predictive control," IEEE Control Syst., vol. 22, no. 1, pp. 44-52, Feb. 2002.

[38] Q. Shafiee, Č. Stefanović, T. Dragičević, P. Petar, C. Juan, Vasquez and M. G. Josep, "Robust networked control scheme for distributed secondary control of islanded microgrids," IEEE Trans. Ind. Electron., vol. 61, no. 10, pp.5363-5374, Oct. 2013.

[39] A. Kulmal, S. Repo, and P. Jarventausta, "Coordinated voltage control in distribution networks including several distributed energy resources," IEEE Trans. Smart Grid, vol. 5, no. 4, pp. 2010-2020, Jul. 2014. 